\title{
EMPLEO EN LA ECONOMÍA INFORMAL: MAYOR AMENAZA QUE LA PANDEMIA DEL COVID-19
}

\author{
Roberto Carlos Dávila Morán* \\ https://orcid.org/0000-0003-3181-8801
}

RECIBIDO: Diciembre 2020 / ACEPTADO: Marzo 2021 / PUBLICADO: Mayo 2021

Como citar: Dávila Morán, Roberto. (2021). Empleo en la economía informal: mayor amenaza que la pandemia del covid-19. Telos: revista de Estudios Interdisciplinarios en Ciencias Sociales, 23 (2), Venezuela. (Pp.403-417).

DOI: www.doi.org/10.36390/telos232.12

\section{RESUMEN}

La pandemia del COVID-19 parece perjudicar de manera desigual a las personas con empleo informal, que con menos frecuencia reciben ayuda del gobierno que las personas con empleo formal. El propósito de este ensayo es realizar un análisis reflexivo sobre empleo en la economía informal en tiempos de pandemia provocada por el COVID 19. La metodología se basa en una investigación documental, en el que se tomaron en consideración diversas fuentes documentales como artículos publicados en revistas científicas e informes de organismos internacionales, con el fin de documentar el problema; entre los principales autores para el desarrollo documental se tienen Williams y Horodnic (2016a, 2016b), ILO (2014), ILO (2015) y Webb et al., (2020). La pandemia tiene efectos importantes a corto y largo plazo para el empleo informal y la economía informal. La pandemia del COVID-19 podría apresurar las tendencias actuales y obligar nuevas soluciones para preservar la seguridad básica del trabajo mientras ayuda a las organizaciones a seguir siendo competitivas. Las políticas gubernamentales que promueven la seguridad laboral de los ingresos, los movimientos hacia la formalización del empleo y la equidad para los empleados informales son peculiarmente considerables. Los resultados proponen que los gobiernos deben tener en cuenta atentamente el apoyo claro a quienes tienen empleos informales para crear estructuras justas, resilientes y éticas para los trabajadores, las industrias, las economías y sociedad en general. Como conclusiones se tiene que la reingeniería de la economía pos pandemia puede conducir a una reconsideración de las prácticas de empleo ampliamente utilizadas que tienden a reducir las condiciones de los trabajadores y la protección de la salud, a fin de obtener una ventaja competitiva.

Palabras clave: COVID-19; empleo informal; empleo formal; economía informal.

* Universidad Privada del Norte (UPN), Perú. Correo electrónico rdavila430@gmail.com 


\title{
Employment in the informal economy: greater threat than the Covid-19 pandemic
}

\begin{abstract}
The COVID-19 pandemic appears to unevenly harm those in informal employment, who are less frequently assisted by the government than those in formal employment. The purpose of this essay is to carry out a reflective analysis on employment in the informal economy in times of the pandemic caused by COVID 19. The methodology is based on a documentary research, in which various documentary sources such as articles published in magazines were taken into consideration scientific reports and reports from international organizations, in order to document the problem; Among the main authors for documentary development are Williams and Horodnic (2016a, 2016b), ILO (2014), ILO (2015) and Webb et al., (2020). The pandemic has important short- and long-term effects on informal employment and the informal economy. The COVID-19 pandemic could accelerate current trends and force new solutions to preserve basic job security while helping organizations remain competitive. Government policies that promote job security of income, movements toward employment formalization, and equity for informal employees are peculiarly significant. The results propose that governments should carefully consider clear support for those in informal jobs to create fair, resilient and ethical structures for workers, industries, economies and society in general. The conclusions are that the reengineering of the post-pandemic economy can lead to a reconsideration of widely used employment practices that tend to reduce the conditions of workers and the protection of health, in order to obtain a competitive advantage.
\end{abstract}

Keywords: COVID-19; informal employment; formal employment; informal economy.

\section{Introducción}

En marzo de 2020, la propagación de la pandemia del coronavirus (COVID-19), llevo a la Organización Mundial de la Salud (OMS) a declarar una emergencia de salud pública mundial. Las respuestas políticas adoptadas por los gobiernos, incluidas las medidas de bloqueo, y la recesión económica resultante afectaron particularmente a ciertos sectores, que utilizan a un gran número de empleados y autónomos en las economías formales e informales. Estos sectores incluían bares y restaurantes, artes, entretenimiento y otros proveedores de ocio, proveedores de transporte, empresas de alojamiento e inmobiliarias, así como agentes de viaje y operadores turísticos (ILO, 2020).

Con partes de muchas economías desarrolladas que aún no se han recuperado por completo, incluso una década después de la crisis financiera de 2008, los gobiernos de todo el mundo se han apresurado a implementar una variedad de enfoques para manejar esta crisis de salud. Aunque la pandemia del COVID-19, parece afectar de manera desproporcionada a las personas con empleos informales, a menudo reciben menos apoyo del gobierno que las personas con empleo formal.

Sigue habiendo incertidumbre sobre la duración y los efectos de esta pandemia, o incluso si las oleadas consiguientes reaparecerán en los meses y años venideros. Su impacto indudablemente llevará a que muchas organizaciones cierren o reduzcan sus operaciones y su desarrollo se verá muy afectado durante muchos años. Un aumento de la carga de la deuda y 
la reducción de los mercados 0 una pérdida sustancial de ingresos experimentada por los empleadores ya ha tenido efectos multiplicadores en cadena en otros, incluidos su personal, proveedores, colaboradores comerciales y comunidades. Por el contrario, para otros empleadores la crisis puede desencadenar un periodo de reinvención y adaptación, que los lleve a prosperar a medida que aprovechan las oportunidades generadas por los cambios en la cadena de suministro y la demanda, las actividades y los hábitos de los consumidores.

En muchos países, como el Reino Unido, los espacios sociales de la economía más afectados por el COVID-19, en sus primeras etapas han sido espacios sociales de bajos salarios que tienden a emplear de manera desproporcionada a jóvenes, mujeres, personas de grupos étnicos y minoritarios (Papoutsaki y Wilson, 2020). Además, muchos trabajadores en estos espacios sociales están empleados de manera informal y no han podido recibir el apoyo financiero gubernamental disponible para los empleados en la economía formal (Williams y Kayaoglu, 2020 ; ITUC, 2020).

En estos países económicamente desarrollados, el empleo informal comprende casi una quinta parte (18\%) de los trabajadores, pero el $61 \%$ de la fuerza laboral mundial (ILO, 2018). A corto plazo, es probable que la pandemia afecte a los trabajadores y solicitantes de empleo con menor protección social y laboral, en particular a los que se encuentran en el mercado laboral informal. Sin embargo, los efectos a largo plazo sobre estos y otros tipos de trabajadores siguen sin estar claros y podrían seguir trayectorias diferentes. Este documento busca explorar algunas de las implicaciones de la pandemia para el empleo en la economía informal, particularmente en países económicamente desarrollados o de altos ingresos.

Como se analiza a continuación, el empleo informal abarca actividades que se consideran ilegales o que no siguen regulaciones laborales y tributarias, pero que son legales o legitimas. En Estados Unidos, Katz y Krueger (2019), descubrieron que el crecimiento del empleo más reciente se originó a partir de acuerdos laborales alternativos que involucraban a trabajadores de agencias de ayuda temporal, trabajadores de guardia, trabajadores contratados de empresas y contratistas independientes o autónomos.

La proporción de estos trabajos aumento del 10,7\% a un rango del $12,6 \%$ al $15,8 \%$ de los trabajos de 2005 a 2015. Dado que la mayoría de las relaciones laborales informales están asociadas con un empleo de baja calidad y una protección social insuficiente, esta tendencia dificulta la acción colectiva para mejorar las condiciones de trabajo. Parece que la pandemia puede haber acelerado una tendencia existente hacia una mayor proliferación de trabajos no tradicionales que incluye condiciones de trabajo justas, igualdad de oportunidades y protección social (European Commission, 2017).

A partir de lo anterior, se planteó el presente trabajo donde se muestran los hallazgos sobre documentos publicados en revistas científicas (indexadas en bases de datos como Scopus y Web of Science), y en portales de organismos internacionales como la Organización Internacional del Trabajo (OIT), la Comisión Económica para América Latina y el Caribe (CEPAL), la Organización de las Naciones Unidas para la Alimentación y la Agricultura (FAO), entre otras, donde se revisaron un total de 200 documentos de los cuales 22 cumplieron con el criterio de estudio del impacto del COVID-19, sobre el empleo informal, el cual fue el criterio de inclusión. Adicionalmente se incluyeron 23 documentos donde se trató la problemática del empleo informal, los cuales sustentaron el problema y sirvieron para ubicar la investigación dentro del contexto específico. 


\section{¿Quién se relaciona con la economía informal y por qué?}

Los empleadores y los trabajadores individuales tienen diferentes razones para comprometerse con la economía informal. Los motivos de los empleadores surgen principalmente de la competencia, las limitaciones financieras, la falta de conciencia y la comprensión inadecuada del cumplimiento o una decisión deliberada de no cumplir (ILO, 2015). Por lo tanto, es claro que las actividades económicas en la economía informal pueden ocultarse a las autoridades e instituciones no solo para obtener ganancias financieras, sino también para evitar responsabilidades regulatorias (Medina y Schneider, 2018 ; Williams, 2011).

Esto incluye actividades empresariales en las primeras etapas del desarrollo 0 comercialización de productos cuando se trata de un mayor riesgo de fracaso y pérdida financiera (Williams y Martinez, 2014). Por ejemplo, la evidencia del Reino Unido sugiere cuando comienzan su negocio para probar la viabilidad de su producto o servicio. Si bien los empleadores pueden tener diferentes razones para participar en la economía informal, generalmente surgen de las reglas de competencia globales, locales y las limitaciones financieras (ILO, 2015).

La separación entre economías formales e informales suele ser indistinta. Dado que la naturaleza del proceso empresarial se centra en el reconocimiento y la explotación de oportunidades comerciales, las decisiones relativas al crecimiento a menudo conducen a actividades en la periferia o directamente en la economía informal (Webb et al., 2009). Este parece ser el caso particularmente de los emprendedores orientados a hacer crecer sus empresas en lugar de aquellos que buscan un estilo de vida determinado o solo complementan sus ingresos.

Usando la teoría de la legitimidad, una percepción o suposición generalizada de que las acciones de una entidad son deseables, adecuadas o apropiadas dentro de algún sistema socialmente construido de normas, valores, creencias y definiciones, sugiere que las definiciones de los empresarios de una oportunidad y la valoración de lo que constituye una acción legitima en la economía informal serán diferentes. Tal valoración, por ejemplo, podría llevar a la decisión de utilizar trabajadores indocumentados o no declarados (por ejemplo, inmigrantes ilegales) para producir productos, actividades legales y legítimos como la construcción de viviendas, la producción agrícola o los servicios domésticos (Raijman, 2001) ; o productos usualmente legítimos pero ilegales como música pirateada y artículos falsificados (Givon et al., 1995).

Es especialmente importante reconocer que el compromiso en la economía informal no siempre es una elección individual, sino más bien una consecuencia de las instituciones y prácticas informales, así como de los tipos de trabajo que realizan las personas; por ejemplo, temporal y trabajos de temporada que son comunes en la agricultura de subsistencia y otros trabajos agrícolas, hortícolas y forestales, servicios domésticos, cuidado, restauración y el turismo, el comercio minorista (Suchman, 1995). Como se discutió anteriormente, estos trabajos temporales / estacionales se caracterizan por la ausencia de contratos y corta duración. Se trata de sectores predominantemente de baja tecnología y de uso intensivo de mano de obra que emplean trabajadores pocos calificados (Schneider, 2011).

Los factores determinantes de la economía informal han sido objeto de estudio en los últimos tiempos en diferentes partes del mundo, debido a que como se mencionó, una fracción 
importante de la población mundial trabaja de forma informal, al respecto Angel-Urdinola y Tanabe (2012), demostraron que en países africanos el empleo informal está relacionado con el tamaño del sector público, las principales actividades económicas de los países y la edad de las personas, por lo que indicaron que la inversión pública es fundamental para promover el empleo formal y disminuir la informalidad.

Estos mismos determinantes fueron reportados por Thapa (2014), al analizar el problema del empleo informal en Nepal, e introdujeron otro determinante, el aspecto étnico lo que es indicativo de que en países más pobres, ciertos grupos sociales tienen mayor tendencia a pertenecer al sector económico informal. En otra investigación realizada en Pakistán (Williams et al., 2016), concluyeron que el empleo informal está relacionado con las características de los empresarios y las empresas, además indicaron que el nivel de estudios influye de manera importante en la tendencia de las personas a ingresar al sistema económico informal.

Es claro que el empleo informal, representa una seria amenaza para el control de situaciones de emergencia como las presentadas a raíz de la pandemia producida por el COVID-19, ya que como lo advierte la Organización Internacional del Trabajo (OIT, 2020), las personas que se dedican a la economía informal representan el $62 \%$ de la fuerza de trabajo a nivel mundial y los mismos al no depender de rentas ni ahorros no pueden dejar de trabajar y quedarse en sus casas debido a que sus ingresos económicos son del día a día, lo que ha sido un problema sobre todo en los países de bajos ingresos donde el empleo informal representa aproximadamente el $90 \%$ de la fuerza laboral, algo que también es crítico en países con ingresos medios ( $67 \%$ de empleo informal).

En este mismo aspecto, (Weller, 2020), indica que la pandemia del COVID-19 amenaza con producir un aumento en el porcentaje de trabajadores informales a nivel mundial, debido a que una proporción considerable de profesionales que están dentro del sector formal, al perder sus empleos deben insertarse a sectores informales de menor productividad. La Organización de las Naciones Unidas para la Alimentación y la Agricultura (FAO, 2020), también indica que los sectores relacionados con la agricultura, sobre todo en ámbitos rurales, son los más vulnerables ante la pandemia del COVID-19 debido a que los trabajadores son en su mayoría asalariados.

\section{Participación y compromiso de la economía informal}

Williams y Horodnic (2016a), estableció tres teorías en competencia. Primero, la teoría de la modernización explica la economía informal en términos de la falta de desarrollo económico y modernización de las burocracias estatales; segundo, los enfoques neoliberales sugieren que la participación en la economía informal es una respuesta a reglas extensas, regulaciones onerosas y altos impuestos percibidos; mientras que en tercer lugar, la teoría de la economía política sostiene que la economía informal es el resultado de una intervención estatal inadecuada y una falta de protección para los trabajadores (ILO, 2014).

Estos enfoques, si bien son útiles para hacer distinciones entre diferentes contextos nacionales y expectativas de una relación ideal entre el estado, la regulación y las actividades económicas, han sido criticados por no reconocer las motivaciones intrínsecas específicas de los actores individuales que eligen, o no, participar en el proceso. Algunos actores pueden optar por el empleo informal cuando la recompensa es mayor que el costo esperado, por 
ejemplo, de ser capturados o castigados, o cuando las alternativas son peores (por ejemplo, desempleo); otros pueden querer optar por participar incluso si los costos superan los beneficios (Murphy, 2005). Sin embargo, el poder explicativo de tales distinciones entre comportamientos económicos racionales e irracionales como motivadores para el compromiso con la economía informal es limitado.

La teoría institucional y la noción de moral fiscal han propuesto una comprensión alternativa de los comportamientos variados de los actores individuales y las poblaciones (McKerchar et al., 2013). Sugiere diferencias en los niveles de aceptabilidad con respecto a la participación en la economía informal como resultado de la asimetría entre las leyes y regulaciones codificadas de las instituciones formales de una sociedad (moralidad del gobierno o moral del estado) y las normas, valores y creencias no escritas socialmente compartidas de la sociedad que constituyen sus instituciones informales (moral social o moral cívica) (Williams y Horodnic, 2016a). Los valores, normas y creencias de las instituciones informales de una sociedad pueden complementar los de las instituciones formales o sustituir sus reglas si se produce una incompatibilidad con las instituciones formales.

Dos proposiciones clave de la teoría institucional, ayudan a comprender la dinámica entre las economías formal e informal. Primero, la simetría entre las instituciones formales e informales elimina la necesidad de actividades en la economía informal. En segundo lugar, la asimetría entre las instituciones formales de una sociedad y sus instituciones informales, por ejemplo, debido a la falta de confianza en el gobierno, alimenta las actividades de la economía informal. Por lo tanto, cuanto mayor es la asimetría entre la moralidad del gobierno y la moralidad de la sociedad, mayor es la propensión para participar en la economía informal (Williams y Horodnic, 2016a). En otras palabras, esto significa que cuanto menor es el nivel de moral tributaria, mayor es la asimetría y el nivel de participación en la economía informal. Cuanto mayor es la moral fiscal, por ejemplo, debido a una mayor intervención estatal en forma de impuestos más altos que resultan en un mayor gasto y beneficios sociales, menor es la asimetría y el nivel de participación en la economía informal.

Los hallazgos en una variedad de contextos geográficos, sugieren que la moral fiscal suele ser más baja entre los hombres, las personas solteras, los desempleados y los trabajadores independientes, pero aumenta con la religiosidad, la edad, el estatus social percibido, los ingresos y se relaciona negativamente con los años pasados en educación (Williams y Martinez, 2014). Estos conocimientos pueden ser útiles para planificar intervenciones centradas en abordar la economía informal y apoyar la transición de los trabajadores del empleo informal al formal.

Williams y Horodnic (2016b), argumentan que es posible reducir la participación en la economía informal mediante el uso de intervenciones como: mejorar el nivel educativo, las personas mayores asesorando a los más jóvenes y mejorando la participación de las mujeres en el mercado laboral. La pandemia del COVID-19, puede alterar los juicios relacionados con la moral fiscal y la gente comienza a reconsiderar el valor positivo y los beneficios de los impuestos para la salud y el apoyo al empleo derivados de la economía formal (Webb et al., 2020).

Sin embargo, una visión alternativa (especialmente en lugar donde las respuestas gubernamentales han sido deficientes) podría ser que las personas están más dispuestas a evitar el sector formal, ya que sentirán que necesitan invertir en su propia resiliencia personal 
en lugar de depender de una intervención gubernamental inadecuada. La concepción de respuestas gubernamentales adecuadas a los juicios individuales sobre el equilibrio costobeneficio es especialmente pertinente para los sectores que generalmente son difíciles de regular, como lo servicios personales y domésticos en España, por ejemplo, la asociación SEDOAC (Servicio Doméstico Activo) que representa a los trabajadores domésticos y del cuidado insto al gobierno a extender las medidas de apoyo financiero relacionadas con el COVID-19, (por ejemplo, exenciones fiscales o esquemas de ingresos para hogares vulnerables) y aquellos trabajadores no incluidos en el sistema general de seguridad social (WIEGO, 2020). Del mismo modo, académicos y activistas en Italia publicaron una petición llamando la atención sobre la exclusión de los trabajadores domésticos y de cuidados del esquema de apoyo del gobierno llamado Cura Italia, situación que fue destacada por Alemani et al., (2020).

En este mismo aspecto, Narula (2020), propuso que la crisis en el empleo informal generado por el COVID-19, debe crear las circunstancias para un mayor compromiso activo de los gobiernos con los sectores económicos informales, de manera que la situación a las empresas informales debe ser llevado al mismo nivel que las empresas formales dentro de la política industrial. El autor citado, además destaca el papel del Estado como determinante crucial en la búsqueda de socios, creando incentivos para los sectores informales de forma sistemática, y para reducir los costes de transacción en dicho compromiso, lo que podría aportar beneficios a largo plazo, aunque resulten costosas a corto plazo.

Es claro que, ante la informalidad del empleo, las políticas gubernamentales son de vital importancia en la reducción del impacto del COVID-19, en este sector de la economía, lo cual es enfatizado por Parvathamma (2020), quien al analizar la situación del empleo ante el COVID-19, en la India, indicó que en un país donde la mano de obra migrante informal/no organizada representa casi el $70 \%$ de la mano de obra, la misma se ve gravemente afectada por la pérdida de empleo, por lo que la actuación del gobierno es fundamentar para acelerar la recuperación, lo que incluye reforzar la "red de seguridad" de forma significativa para los más vulnerables, permitir la supervivencia de las pequeñas y medianas empresas, reactivar la economía rural y proporcionar ayuda específica a los sectores de riesgo.

\section{Reducir la dependencia de la economía informal}

Como ya se ha comentado, la participación en actividades económicas informales tiene múltiples consecuencias negativas para las personas, la economía formal y las sociedades. Provoca pérdidas fiscales importantes, lo que reduce los ingresos públicos y, por tanto, los ingresos disponibles para servicios y apoyo públicos clave, incluida la protección social; también contribuye a peores condiciones de trabajo y competencia desleal de negocios legítimos, debilitando los sindicatos y la negociación colectiva (ILO, 2014).

Según datos de la Unión Europea y basándose en dos mecanismos básicos de la teoría institucional para abordar la asimetría institucional, Williams y Horodnic (2016, a, b), proponen abordar el problema mediante cambios en las instituciones formales e informales, algo que se acentúa por el efecto de la pandemia del COVID-19, tal como lo indica Zikos (2020) quien sugiere que se debe reunir los resultados de diferentes entornos y las propuestas de diferentes disciplinas de forma sintetizada, para poder tener una visión holística y comprender mejor los procesos de cambio institucional, lo que a su vez, permitiría introducir y 
aplicar políticas con objetivos concretos. Por su parte Alfaro et al., (2020), consideran que los gobiernos deben asumir una gran responsabilidad en los procesos de cambio generados como consecuencia de la pandemia del COVID-19, sobre todo en economías emergentes y en desarrollo ya que la capacidad para mitigar los impactos económicos son más limitadas y la capacidad para hacer cumplir los cierres es más débil.

El primer mecanismo se basa en la idea de desincentivos (sanciones y penas) para prevenir actividades socialmente legítimas, pero ilegales, por ejemplo, comunicando y mejorando la probabilidad de detención mediante inspección (Hasseldine et al., 2007). Sin embargo, este enfoque puede ser contraproducente, ya que puede socavar el respeto por la equidad del sistema y, por lo tanto, reducir el cumplimiento voluntario, lo que lleva a una mayor participación en la economía informal (Murphy, 2005). En países como Corea del Sur, aun cuando se aplicaron sanciones y penalidades para los casos en los que se violaran las medidas adoptadas por el gobierno durante la pandemia del COVID-19, las mismas no surtieron el efecto deseado, por lo que no hubo disminución en los casos de violación a las medidas (Ryu et al., 2020).

El segundo mecanismo, destaca la necesidad de intensificar los comportamientos y actividades legales deseables (Mathias et al., 2015), por ejemplo, mediante incentivos fiscales directos e indirectos. Este segundo mecanismo ha sido adoptado por la mayoría de los gobiernos de los países a nivel mundial (CEPAL, 2020), y en algunos casos por las propias empresas privadas grandes, como el caso de España donde varias empresas han mostrado un gran compromiso con la sociedad, desarrollando acciones que palian las consecuencias de la COVID-19 como otras han desarrollado varias estrategias con diferentes objetivos (GarcíaSánchez y García-Sánchez, 2020). Este enfoque, sin embargo, no resuelve por completo la cuestión del cumplimiento y la moralidad.

\section{Consideraciones y conclusiones}

Este documento considero definiciones relacionadas con la economía informal, por qué personas y organizaciones podrían participar en ella y reflexionó sobre algunos efectos de la pandemia del COVID-19, en estos trabajadores. Es demasiado pronto para identificar los efectos a medio y largo plazo de la pandemia, ya que el riesgo de múltiples oleadas de infección y la disponibilidad de tratamientos médicos eficaces, como las vacunas, sigue sin conocerse. Sin embargo, está claro que incluso en las economías de altos ingresos, las formas de participación y las razones para participar en actividades económicas informales varían entre las diferentes poblaciones y contextos socioeconómicos, como lo mencionan Webb et al., (2020).

La distribución de poder entre empleadores y trabajadores en el mercado laboral mundial se ha debatido ampliamente y el deterioro de las condiciones de trabajo y remuneración está cada vez más en la agenda de los sindicatos y otras agencias de promoción. Así mismo, la creciente urbanización ha provocado un crecimiento paralelo de las economías informales, que desempeñan un importante papel en el alivio del desempleo y la pobreza en las ciudades y el estudio de este fenómeno no pueden ser explicadas completamente por cada una de las principales teorías de la informalidad que se tienen en la actualidad (Huang et al., 2020). 
La extrema reducción de puestos de trabajo en 2020, y la alta incertidumbre sobre el futuro debido a la pandemia, ha modificado fundamentalmente el equilibrio de costos y beneficios relacionados con el empleo informal. En particular, puede aumentar los costos de las personas si las oportunidades de empleo formal o informal disminuyen rápidamente y el acceso de apoyo al empleo del gobierno no está disponible, a diferencia de los de la economía formal. También hay efectos psicológicos y socioeconómicos más amplios en las personas, las comunidades y las regiones, lo que sugiere que los problemas de la disponibilidad de empleo, la flexibilidad y la seguridad seguirán siendo importantes. Lo anterior, es clave en el proceso de recuperación económica de los países, lo que lleva a los gobiernos a aplicar políticas de subsidio al empleo, que pueden incentivar a las empresas a preservar el empleo, lo que según Carranza et al., (2020), estas políticas que apoyan la liquidez de las empresas lo hacen con poca eficacia.

Por el contrario, la pandemia puede alentar a algunos empleadores a alejarse del trabajo formal, debido a las oportunidades para una respuesta más flexible y rápida a los grandes choques (como la pandemia) que permiten los acuerdos informales. Para reforzar esto, las pequeñas y medianas empresas, donde se concentran los trabajos informales, también pueden sufrir desproporcionadamente las cadenas de suministro interrumpidas: las empresas más grandes pueden tener preferencia por bienes con una oferta limitada, dejando a las más pequeñas incapaces de producir de manera eficiente, o las cadenas de suministro pueden ser fundamentalmente alterado debido a la pandemia. Es claro, que la pequeña empresa se ha visto afectada en mayor proporción debido a la informalidad de la mayoría de sus operaciones, tal como lo analizaron Bartik et al., (2020).

Sin embargo, es probable que un resultado sea una mayor tensión no resuelta entre el deseo de los trabajadores informales de obtener mayores ingresos y seguridad en el empleo, por un lado, $y$, por el otro, la lucha de algunos empleadores por una flexibilidad laboral continua frente a los cambios laborales inducidos por la pandemia y demandas de los clientes. El contraste entre las perspectivas del empleador y el trabajador, se vuelve particularmente sobresaliente en el intento de los empleadores de transferir el riesgo / costo de tal flexibilidad al gobierno y los trabajadores. Por lo tanto, es crucial considerar como la política gubernamental, incluida la regulación del mercado laboral y el apoyo al empleo continuo durante la pandemia, puede mediar entre los diferentes actores a largo plazo.

Surge la pregunta de cómo se puede incentivar a los empleadores para que se retiren de las practicas informales y cómo se puede restablecer el equilibrio en el intercambio de trabajo para construir bases más sólidas para actividades resilientes, justas, éticas, legales y legitimas en el mercado laboral. Como Williams y Horodnic (2016a), sugieren que las intervenciones prospectivas tienen muchas más posibilidades de ser eficaces si consideran la dinámica de la moral tributaria $\mathrm{y}$, simultáneamente, abordan los cambios necesarios para fortalecer las instituciones formales e informales. De manera optimista, podría ser que, en esta pandemia mundial, el diseño e implementación de medidas inclusivas de apoyo al empleo y al bienestar aumente y refuerce los principios de equidad y cuidado (Fairlie, 2020). La propagación de este proceso podría transformar las actitudes y comportamientos en las instituciones informales y, por lo tanto, fomentar una mayor confianza, creencia y compromiso con las reglas y regulaciones en la economía formal. 
El empleo formal e informal está estrechamente vinculado a través de la completa interacción de intercambios entre instituciones formales e informales y otros actores. Cuando no se cumplen las regulaciones formales de empleo, puede comenzar el trabajo de carácter informal, lo que puede desdibujar los límites entre estos dos contextos. Al centrarse en la perspectiva de los actores individuales (tanto empleados como empleadores), que participan en la economía informal, sus decisiones y acciones pueden regirse por diferentes racionalidades, que a menudo surgen de las incompatibilidades y contradicciones dentro de los sistemas de empleo.

Por lo tanto, sería interesante explorar más a fondo la conexión entre la economía informal y un sistema más amplio de seguridad social y bienestar dentro de contextos nacionales e internacionales específicos que claramente surgieron durante la pandemia. Además, este documento sugiere que los gobiernos deben considerar cuidadosamente el apoyo a las personas con empleos informales, incluida su incorporación a empleos más formales con mayor seguridad, protección y con las contribuciones asociadas a los impuestos y la seguridad social. Tal como lo mencionaron Pitoyo et al., (2020), la crisis como consecuencia de esta pandemia supone una nueva experiencia para el sector informal a la hora de afrontar la crisis. El sector informal no es siempre un héroe durante la crisis, sino un sector alternativo que ofrece flexibilidad empresarial.

La reingeniería de la economía pandémica posterior al COVID-19, puede conducir a una reconsideración de las prácticas de empleo ampliamente utilizadas que tienden a reducir las condiciones de los trabajadores y la protección de la salud, a fin de obtener una ventaja competitiva. Por un lado, el aumento en las últimas décadas de una versión moderna de la economía de los trabajos por encargo (personas que trabajan en base a trabajos de corta duración, a menudo autónomos) puede aumentar con un mayor deseo de flexibilidad entre los empleadores. Lo más probable, es que su aversión a contratar personal se vea agravada por la posibilidad de futuros trastornos económicos y de salud. Por otro lado, puede haber efectos contrarios a medida que los trabajadores busquen una mayor seguridad laboral, sanitaria y una red de protección contra el desempleo más eficaz.

Es probable que el aumento del trabajo a domicilio y las operaciones a distancia debido a la pandemia acelere las tendencias en los próximos años, alterando potencialmente el equilibrio entre el empleo formal e informal. El aumento de las tendencias de compra en línea, acelerado por la pandemia, puede aumentar la subcontratación informal de trabajo. Particularmente en tiempos de crisis a largo plazo, es posible que el empleo formalizado se vuelva más informal y deje de ser un proveedor de condiciones laborales significativamente mejores que los empleos informales (Shapland y Heyes, 2017).

Se necesitan más investigaciones sobre cómo se resuelven las tensiones potenciales en la económica informal entre trabajadores, gobiernos, empleadores y otras instituciones en diferentes contextos. Si bien los efectos a corto plazo de la pandemia del COVID-19, son complejos y poco claros, es probable que los efectos a largo plazo sobre el empleo (formal e informal) y sobre diferentes grupos de la sociedad sean considerables, afectando potencialmente la polarización económica; por lo que se requiere un análisis exhaustivo de los posibles impactos en diferentes grupos demográficos, étnicos, género y otros. La investigación también debe considerar el desarrollo de los efectos distributivos del empleo informal y las implicaciones de estos para nuestra comprensión teórica del empleo en general. 


\section{Referencias Bibliograficas}

Alemani, Claudia; Amorosi, Lucia; Busi, Beatrice; Maioni, Raffaella; Marchetti, Sabrina; Sarti, Raffaella; Turrini, Olga; Vianello, Francesca Alice; Zucca, Gianfranco. (2020). Towards a Caring Democracy: An Appeal Published by a Group of Italian Researchers. Extraido de: https://www.worck.eu/2020/04/13/towards-a-caringdemocracyl

Alfaro, Lauro; Becerra, Oscar; Eslava, Marcela. (2020). EMES and COVID-19: Shutting down in a world of informal and tiny firms. National Bureau of Economic Research. Extraido de: http://www.nber.org/papers/w27360

Angel-Urdinola, Diego; Tanabe, Kimie. (2012). Micro-Determinants of Informal Employment in The Middle East and North Africa Region. Washington, D.C: The World Bank. Extraido de:

https://openknowledge.worldbank.org/bitstream/handle/10986/26828/665940NWP00

PUB0Box365795B0SPDP01201.pdf?sequence=1

Bartik, Alexander; Bertrand, Marianne; Cullen, Zoe; Glaeser, Edward; Luca, Michael; Stanton, Christopher. (2020). The impact of COVID-19 on small business outcomes and expectations. Proceedings of the National Academy of Sciences, 117(30), United States. (Pp. 17656-17666). https://doi.org/10.1073/pnas.2006991117

Carranza, Eliana; Farole, Thomas; Gentilini, Ugo; Morgandi, Matteo; Packard, Truman; Santos, Indhira; Weber, Michael. (2020). Gestión de los Impactos de la Crisis del Covid-19 en el Empleo: Opciones de Política para el Alivio y la Reestructuración. Washington, DC: Banco Mundial. Extraido de: https://openknowledge.worldbank.org/bitstream/handle/10986/34263/Managing-theEmployment-Impacts-of-the-COVID-19-Crisis-Policy-Options-for-Relief-andRestructuring-SP.pdf?sequence $=6$ \&isAllowed=y

CEPAL. (2020). Sectores y empresas frente al COVID-19: emergencia y reactivación. Extraido de: https://www.cepal.org/sites/defaultfiles/publication/files/45734/S2000438_es.pdf European Commission. (2017). The European pillar of social rights in, 20 principles. Extraido de: https://ec.europa.eu/commission/priorities/deeper-and-fairer-economic-andmonetary-union/european-pillar-social-rights/european-pillar-social-rights-20principles_en\#chapter-i-equal-opportunities-and-access-to-the-labour-market

Fairlie, Robert. (2020). The impact of COVID-19 on small business owners: Evidence from the first 3 months after widespread social-distancing restrictions. Journal of Economics \& Management Strategy, 29(4), United Kingdom. (Pp. 727-740). https://doi.org/10.1111/jems. 12400

FAO. (2020). Repercusiones de la enfermedad por coronavirus (COVID-19) en los trabajadores informales. Roma: Naciones Unidas. https://doi.org/10.4060/ca8560es 
García-Sánchez, Isabel-María; García-Sánchez, Alejandra. (2020). Corporate Social Responsibility during COVID-19 Pandemic. Journal of Open Innovation: Technology, Market, and Complexity, 6(4), Switzerland. (Pp. 126). http://dx.doi.org/10.3390/joitmc6040126

Givon, Moshe; Mahajan, Vijay; Muller, Eitan. (1995). Software piracy: Estimation of lost sales and the impact on software diffusion. Journal of Marketing, 59(1), United States. (Pp. 29-37). https://doi.org/10.1177/002224299505900103

Hasseldine, John; Hite, Peggy; James, Simon; Toumi, Marika. (2007). Persuasive communications: Tax compliance enforcement strategies for sole proprietors. Contemporary Accounting Research, 24(1), United States. (Pp. 171-194). https://doi.org/10.1506/P207-004L-4205-7NX0

Huang, Gengzhi; Xue, Desheng; Wang, Bo. (2020). Integrating Theories on Informal Economies: An Examination of Causes of Urban Informal Economies in China. Sustainability, $\quad 12, \quad$ Switzerland. (Pp. 2738-2754). http://dx.doi.org/10.3390/su12072738

ILO. (2014). Transitioning from the Informal to the Formal Economy. Extraido de: https://www.ilo.org/wcmsp5/groups/public/---ed_norm/--relconf/documents/meetingdocument/wcms_218128.pdf

ILO. (2015). World Employment and Social Outlook 2015: The changing nature of jobs. Extraido de: https://www.ilo.org/wcmsp5/groups/public/---dgreports/---dcomm/--publ/documents/publication/wcms_368626.pdf

ILO. (2018). Women and Men in the Informal Economy: A Statistical Picture. Extraido de: https://www.ilo.org/global/publications/books/WCMS_626831/lang--en/index.htm

ILO. (2020). COVID-19 and the World of Work: Impact and Policy Responses. Extraido de: https://www.ilo.org/wcmsp5/groups/public/---dgreports/--dcomm/documents/briefingnote/wcms_738753.pdf

ITUC. (2020). Putting people first: 12 governments show the world how to protect lives, jobs and incomes. Extraido de: https://www.ituc-csi.org/putting-people-first?lang=en

Katz, Lawrence; Krueger, Alan. (2019). The rise and nature of alternative work arrangements in the United States, 1995-2015. ILR Review, 72(2), United States. (Pp. 382-416). https://doi.org/10.1177/0019793918820008

Mathias, Blake; Lux, Sean; Crook, Russell; Autry, Chad; Zaretzki, Russell. (2015). Competing against the unknown: the impact of enabling and constraining institutions on the informal economy. Journal of Business Ethics, 127(2), Netherlands. (Pp. 251-264). https://doi.org/10.1007/s10551-013-2030-6

McKerchar, Margaret; Bloomquist, Kim; Pope, Jeff. (2013). Indicators of tax morale: an exploratory study. eJTR, 11(1), Bulgaria. (Pp. 5-22). Extraido de: 
https://heinonline.org/HOL/LandingPage?handle=hein.journals/ejotaxrs11\&div=5\&id= \&page $=$

Medina, Leandro; Schneider, Friedrich. (2018). Shadow economies around the world: what did we learn over the last 20 years? Extraido de:

https://papers.ssrn.com/sol3/papers.cfm?abstract_id $=3124402$

Murphy, Kristina. (2005). Regulating more effectively: the relationship between procedural justice, legitimacy and tax non-compliance. Journal of Law and Society, 32(4), United Kingdom. (Pp. 562-589). https://doi.org/10.1111/j.1467-6478.2005.00338.x

Narula, Rajneesh. (2020). Policy opportunities and challenges from the COVID-19 pandemic for economies with large informal sectors. Journal of International Business Policy, 3(3), United States. (Pp. 302-310). https://doi.org/10.1057/s42214-020-00059-5

OIT. (2020). La crisis de COVID-19 y la economía informal. Respuestas inmediatas y desafíos de política. Ginebra: Organización Internacional del Trabajo. Extraido de: https://www.ilo.org/wcmsp5/groups/public/---ed_protect/---protrav/--travail/documents/briefingnote/wcms_745450.pdf

Papoutsaki, Dafni; Wilson, Tony. (2020). Covid-19 and the low paid: Early analysis of Labour Force Survey. Extraido de: https://www.employmentstudies.co.uk/system/files/resources/files/IES\%20briefing\%20-\%20Covid19\%20and\%20the\%20low\%20paid\%20FINAL2_0.pdf

Parvathamma, Gaura. (2020). Unemployment dimensions of COVID-19 and Government response in India -An analytical study. International Journal of Health and Economic Development, 6(2), United States. (Pp. 28-35). Extraido de: https://search.proquest.com/openview/d8d4a566ea324a209e7326d486ee5ac0/1?pqorigsite $=$ gscholar $\& \mathrm{cbl}=2037330$

Pitoyo, Agus; Aditya, Bagas; Amri, Ikhwan. (2020). The impacts of COVID-19 pandemic to informal economic sector in Indonesia: Theoretical and empirical comparison. E3S Web of Conferences, 200, 03014. https://doi.org/10.1051/e3sconf/202020003014

Raijman, Rebeca. (2001). Mexican immigrants and informal self-employment in Chicago. Human organization, 60(1), United States. (Pp. 47-55). https://doi.org/10.17730/humo.60.1.emtq4bq4c70tqyqr

Ryu, Sukhyun; Hwang, Youngsik; Yoon, Hongbi; Chun, Byung. (2020). Self-Quarantine Noncompliance During the COVID-19 Pandemic in South Korea. Disaster Medicine and Public Health Preparedness, United Kingdom. (Pp. 1-4). https://doi.org/10.1017/dmp.2020.374

Schneider, Friedrich. (2011). The shadow economy and shadow economy labor force: What do we (not) know? Review World Economics, 12(4), Germany. (Pp. 53-92). Extraido de: https://papers.ssrn.com/sol3/papers.cfm?abstract_id=1867038 
Shapland, Joanna; Heyes, Jason. (2017). How close are formal and informal work? International Journal of Sociology and Social Policy, 37(7/8), United Kingdom. (Pp. 374-386). https://doi.org/10.1108//JSSP-06-2016-0071

Suchman, Mark. (1995). Managing legitimacy: Strategic and institutional approaches. Academy of management review, 20(3), United States. (Pp. 571-610). https://doi.org/10.5465/amr.1995.9508080331

Thapa, Resham. (2014). Determinants of Informal Employment and Wage Differential in Nepal. The Journal of Development and Administrative Studies, 22(1-2), Nepal. (Pp. 3750) https://doi.org/10.3126/jodas.v22i1-2.13463

Webb, Aleksandra; McQuaid, Ronald; Rand, Sigrid. (2020). Employment in the informal economy: implications of the COVID-19 pandemic. International Journal of Sociology and Social Policy, 40(9/10), United Kingdom. (Pp. 1005-1019). https://doi.org/10.1108//JSSP-08-2020-0371

Webb, Justin; Tihanyi, Laszlo; Ireland, Duane; Sirmon, David. (2009). You say illegal, I say legitimate: Entrepreneurship in the informal economy. Academy of Management Review, 34(3), United States. (Pp. 492-510). https://doi.org/10.5465/amr.2009.40632826

Weller, Jürgen. (2020). La pandemia del COVID-19 y su efecto en las tendencias de los mercados laborales. Santiago: CEPAL. Extraido de: https://repositorio.cepal.org/bitstream/handle/11362/45759/1/S2000387_es.pdf

WIEGO. (2020). Informal worker demands during COVID-19 crisis. Extraido de: https://www.wiego.org/informal-worker-demands-during-covid-19-crisis

Williams, Colin. (2011). Reconceptualizing women's and men's undeclared work: some results from a European Union survey. Gender, Work \& Organization, 18(4), United Kingdom. (Pp. 415-437). https://doi.org/10.1111/j.1468-0432.2009.00466.x

Williams, Colin; Horodnic, loana Alexandra. (2016a). An institutional theory of the informal economy: some lessons from the United Kingdom. International Journal of Social Economics, 43(7), United Kingdom. (Pp. 722-738). https://doi.org/10.1108/IJSE-122014-0256

Williams, Colin; Horodnic, loana Alexandra. (2016b). Tackling the undeclared economy in the European Union: an evaluation of the tax morale approach. Industrial Relations Journal, 47(4), United States. (Pp. 332-340). https://doi.org/10.1111/irj.12142

Williams, Colin; Kayaoglu, Aysegul. (2020). COVID-19 and undeclared work: impacts and policy responses in Europe. The Service Industries Journal, 40(13-14), United States. (Pp. 1-18). https://doi.org/10.1080/02642069.2020.1757073

Williams, Colin; Martinez, Alvaro. (2014). Do small business start-ups test-trade in the informal economy? Evidence from a UK survey. International Journal of Entrepreneurship 
and Small Business, 22(1), United Kingdom. (Pp. 1-16). https://doi.org/10.1504//JESB.2014.062127

Williams, Colin; Shahid, Muhammad; Martínez, Alvaro. (2016). Determinants of the Level of Informality of Informal Micro-Enterprises: Some Evidence from the City of Lahore, Pakistan. World Development, 84, United Kingdom. (Pp. 312-325). https://doi.org/10.1016/j.worlddev.2015.09.003

Zikos, Dimitrios. (2020). Revisiting the Role of Institutions in Transformative Contexts: Institutional Change and Conflicts. Sustainability, 12(21), Switzerland. (Pp. 90369048). http://dx.doi.org/10.3390/su12219036 